\title{
A Resource Virtualization Mechanism for Cloud Manufacturing Systems
}

\author{
Ning Liu and Xiaoping Li \\ School of Computer Science and Engineering, Southeast University, \\ Nanjing 211189, P.R. China \\ \{ningliu,xpli\}@seu.edu.cn
}

\begin{abstract}
Virtualization is critical for resource sharing and dynamic allocation in cloud manufacturing, a new service-oriented networked collaborative manufacturing model. In this paper, an effective method is proposed for manufacturing resources \& capabilities virtualization, which contains manufacturing resources modeling and manufacturing cloud services encapsulation. A manufacturing resource virtual description model is built, which includes both nonfunctional and functional features of manufacturing resources. The model provides a comprehensive manufacturing resource view and information for various manufacturing applications. The OWL-S is adapted to an upper level ontology model, according to which manufacturing resources $\&$ capabilities are encapsulated into manufacturing cloud service. The proposed method is applied to the virtualization process of an aerospace company. Effectiveness and efficiency are illustrated for the manufacturing cloud services discovery and management.
\end{abstract}

Keywords: virtualization, manufacturing cloud service, cloud manufacturing, resource integration.

\section{$1 \quad$ Introduction}

Cloud manufacturing, as the manufacturing version of cloud computing, offers a new manufacturing model for collaborative manufacturing. Virtualization is the prerequisite for achieving cloud manufacturing, because it determines the robustness of a cloud manufacturing system [1]. By virtualized and encapsulated as cloud services, manufacturing resources \& capabilities can be shared over the manufacturing cloud platform. It is very important to investigate an effective virtualization method for capturing information of manufacturing resources \& capabilities, enhancing the performance of resource sharing and discovery, reducing the holding cost of inventory, and improving the ROI (Return on Investment) of manufacturing resources.

Compared with the virtualization in cloud computing, the cloud manufacturing virtualization is more challenging due to the following considerations. (1)Besides computing resources, manufacturing resources (such as equipment, materials, human)

The original version of this chapter was revised: The copyright line was incorrect. This has been corrected. The Erratum to this chapter is available at DOI: 10.1007/978-3-642-33068-1_20

M. van Sinderen et al. (Eds.): IWEI 2012, LNBIP 122, pp. 46-59. 2012.

() IFIP International Federation for Information Processing 2012 
with complex features also need to be virtualized in an isomorphic manner. It is hard to establish a comprehensive information model for representing manufacturing resources \& capabilities. (2)Cloud manufacturing represents a dynamic organizational structure. In contrast to static mapping between physical resources and logical resources, cloud manufacturing needs a flexible mapping strategy to avoid resource failure caused by uncertain factors. (3)Resources selection in cloud manufacturing largely depends on the granularity of manufacturing demands. For example, the collaboration among enterprises focuses on resources with large granularity, such as transport capacity, warehouse capacity. On the contrary, the collaboration among stations focuses on resources with fine granularity, such as machining tool, warehouse location. Therefore, it is insufficient to describe simple manufacturing capabilities of a manufacturing resource. It is desired to describe manufacturing capabilities of multiple cooperative manufacturing resources. (4)The current standards (XML/SOAP /WSDL/UDDI/OWL) related to web services and semantic web cannot be applied to manufacturing resources directly, because the meaning and usage of manufacturing services are different from traditional web services. A lot of critical information on manufacturing domain cannot be represented by existing service description methods.

Based on the above analysis, this paper explored manufacturing resource \& capability virtualization from Enterprise Interoperability and Collaboration(COIN) perspective. By describing manufacturing capabilities with multi-granularity, the proposed method provides sufficient information for manufacturing demands coming from different collaboration levels. By extending OWL-S, manufacturing resources and capabilities can be encapsulated as manufacturing cloud services, which contribute to resource sharing and dynamic allocation. The paper is organized as follows. Section 2 presents a comprehensive review on manufacturing resources modeling and service encapsulation. Then a cloud manufacturing virtualization framework is described in Section 3. Based on the proposed framework, an effective method for manufacturing resource \& capability virtualization is introduced in Section 4. Section 5 gives a case study to illustrate the advantages of the proposed method. Finally, conclusions are given in Section 6.

\section{Related Work}

Cloud manufacturing focuses on the flexibility and scalability of cooperation among heterogeneous manufacturing resources. Manufacturing resource modeling and manufacturing service description are the critical issues for cloud manufacturing virtualization.

\subsection{Manufacturing Resources Modeling}

Standards as STEP and enhancements of STEP were recognized to play an important role in manufacturing resources modeling [2]. However, the standards only focused on products while overlooked the manufacturing equipment. Vichare et al [3] proposed a unified manufacturing resource model(UMRM), which is based on 
modeling kinematic chains of machines and is more concentrated on geometric aspects of the system. Compared with an assembly system composed of multiple individual devices, UMRM is not adequate to describe the capability of the system. Shen et al [4] provided an agent-based service-oriented integration architecture for collaborative intelligent manufacturing. A unique property of the proposed approach is that the scheduling process of an order is orchestrated on the Internet through the negotiation among agent-based web services.

In the process of product development, manufacturing capabilities determine whether manufacturing resources can reach requirements of product design. Therefore, the representation of various manufacturing capabilities is necessary in virtualization. Liang et al [5] defined resource element as the similar and exclusive capabilities of manufacturing resources, and discussed resource element modeling approach in detail. The proposed method can represent the multi-functional resource closely, reduce the complexity of manufacturing system and improve the agility and flexibility of manufacturing systems. Zhang et al [6] presented a multi-perspective modeling approach to systematically manage distributed manufacturing knowledge on the semantic web. By integrating the industrial, software engineering techniques into ontology-based knowledge engineering process, the proposed approach facilitates the implementation of computer supported cooperative work in distributed manufacturing for semantic web application. These methods can be introduced to model cloud manufacturing resources.

\subsection{Manufacturing Service Description}

Web service technologies are widely used in service-oriented computing. Services description, discovery and integration are based on a series of standards(XML, SOAP, WSDL, UDDI). To improve the resource sharing in a manufacturing grid environment, Shi et al [7] encapsulated resource information by employing XML schema and defined all accessing operations to resources by using WSDL. However, the proposed method is lack of expressing semantic description about manufacturing services.

Ontology and semantic web technology describe web services in a computerinterpretable form, which enables the intelligent interoperation of web services. OWL-S is an ontology-based approach providing a standard for semantic web services. However, OWL-S only addresses the communication capabilities of the services. IOPR-based approaches fall short of logical relationships for the underlying inputs and outputs. Based on an environment ontology, Wang et al [8] built capability specifications of web services. Algorithms for constructing the domain ontology and the matchmaking between the web service capability specifications are presented. The proposed approach can support automated web service discovery. Jang et al [9] defined manufacturing service capability profiles by using OWL and presented a reasoning procedure for matching queries to service description based description logic. The proposed method contributes to intelligent manufacturing service discovery. Ameri et al [10] described manufacturing services formally by manufacturing service description language(MSDL), which has two major parts. The core is composed of basic classes for manufacturing service description. 
The extension includes a collection of taxonomies, subclasses, and instances built by dispersed users from different communities based on their specific needs. Due to lack of semantic description standard for manufacturing services, the above mentioned methods require human involvement to some extent when services interactions are engaged. As a result, the scalability of manufacturing services is limited and the expected economic value of manufacturing services is reduced.

\section{Cloud Manufacturing Virtualization Framework}

Fig.1 illustrates a cloud manufacturing virtualization framework, which consists three layers, manufacturing resource layer, virtual description layer, and service encapsulation layer.

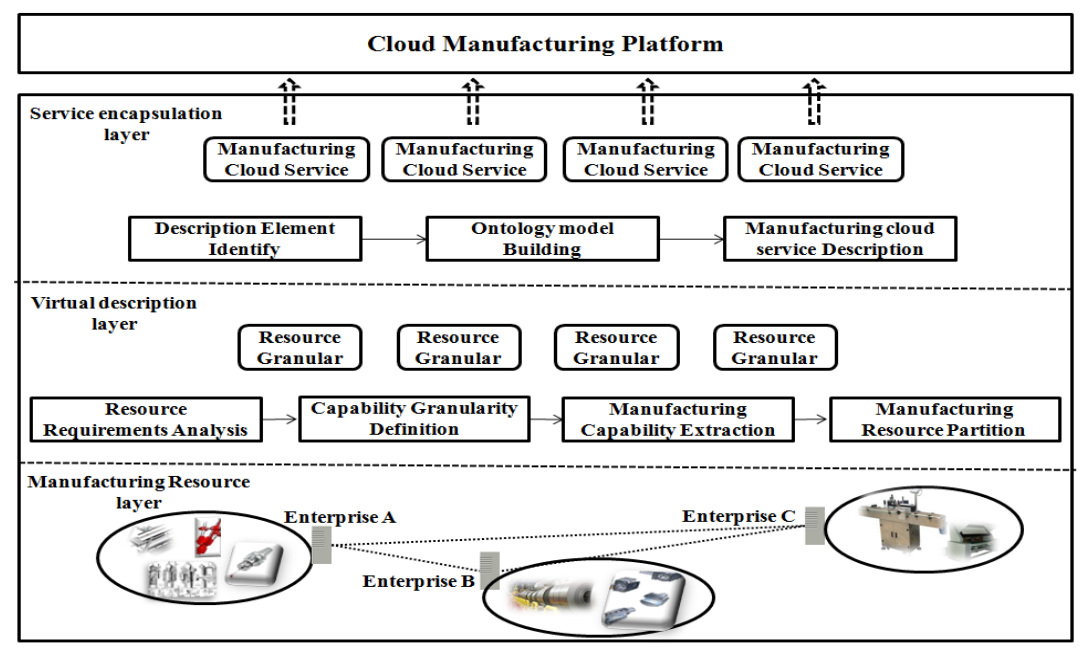

Fig. 1. Cloud manufacturing virtualization framework

In manufacturing resource layer, distributed manufacturing resources can be intelligently sensed and connected into cloud manufacturing systems by using advanced information and communication technologies [11] such as RFID, sensors, GPS. Manufacturing resources refer to various elements to support manufacturing activities throughout product lifecycles. They are the cornerstone of the cloud manufacturing and can be divided into financial resource, technical resource, equipment resource, human resource, software resource, logistics resource, and warehouse resource.

Virtual description layer aims to provide a comprehensive information view for various applications. Manufacturing resource information takes two forms, physical resource information and manufacturing capability information. Physical resource information describes static manufacturing facilities with actual existence. Manufacturing capability information represents an assembly of various operations provided by physical resources. It can be considered as the behavior of the physical 
resource undertaking a particular manufacturing activity with certain constraints. Manufacturing resources can be divided into different results according to manufacturing capabilities with different granularity. Then manufacturing resources with the same or similar capabilities can be aggregated into one granular. An appropriate manufacturing resource model includes both nonfunctional and functional features of manufacturing resources based on a full consideration for both the resource provider and the resource requestor.

Based on ontology modeling approach, manufacturing resources and capabilities are encapsulated as manufacturing cloud services in service encapsulation layer. By this way, manufacturing resources are managed and operated in a service-oriented and flexible way. Furthermore, they can be discovered, selected, and composited in a costeffective manner through the manufacturing cloud platform. Manufacturing cloud service providers are motivated by the profits to be made by charging consumers for accessing services. Manufacturing cloud service consumers request services ranging from all stages of overall product lifecycle. In this paper, a manufacturing cloud service is defined as an encapsulation of a manufacturing resource granular, which may contain one manufacturing resource or many manufacturing resources. The manufacturing cloud service is described with extended OWL-S to implement semantic interoperability among manufacturing enterprises.

\section{$4 \quad$ Proposed Virtualization Method}

\subsection{Multi-granularity Manufacturing Resource Model}

Manufacturing resource information is the digital representation for manufacturing resources. It is the base for various manufacturing applications such as CAD, CAPP, CAM, CAE, ERP etc. In cloud manufacturing, manufacturing resource information is characterized with multi-domain, multi-level, and multi-granularity. The granularity is defined as the measure extent that characterizes the scale or level of problems. Within the field of granular computing, information granularity usually refers to "structural granularity", which signifies the structural abstraction of information items [12]. A structural abstraction can be based on a relatively large information item, such as a document, or its constituent parts. For example, chapters, sections, pages, paragraphs are the different structural abstractions of a book. Similarly, in the process of performing large-scale collaborative manufacturing, complex manufacturing problems are usually recursively decomposed according to the product structure. The matching between complex manufacturing problems and manufacturing resources involves heterogenic system levels (line, cell, station, device), as shown in Fig.2.

Manufacturing capabilities of a physical resource have the characteristics of multiple granularities because a physical resource displays different manufacturing capabilities in different activities and different collaborative demand levels. Within an enterprise, manufacturing resource information with fine granularity is more effective. It is convenient to manage manufacturing resources in a global way. While outside the enterprise, manufacturing resource information with coarse granularity is more popular. It is conductive to release manufacturing resources in an understandable way. 
Therefore, product structure is the basis for measuring manufacturing resources and manufacturing demands. Flexible and effective matching of manufacturing resources against manufacturing demands requires formalized and structured description of the manufacturing capabilities and constraints with multi-granularity.

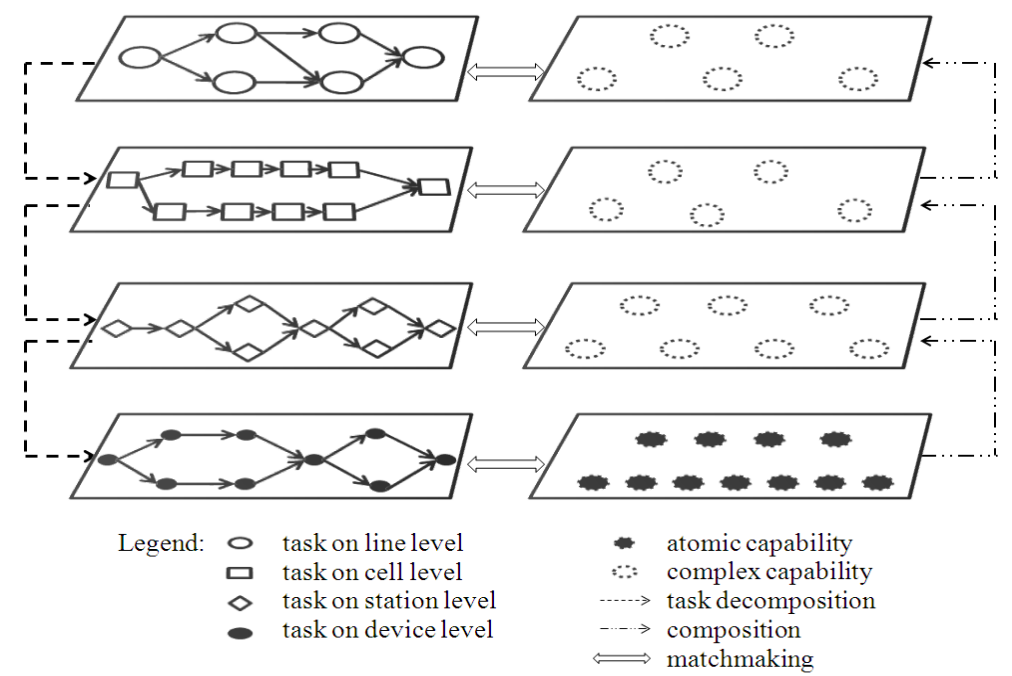

Fig. 2. Multi-granularity matching between manufacturing task and manufacturing capabilities

Definition 1. Let $M R$ be the collection of manufacturing resources, which are connected in cloud manufacturing systems. Let $g$ be a equivalence relation over $M R$. If $g: M R \rightarrow 2^{|M R|}$, then $M R=\bigcup_{i=1}^{n} B_{i}$ and $\left\{B_{j}\right\}$ is a division of $M R . B_{j}$ is called a manufacturing resource granular. $\operatorname{Gr}\left(\left\{B_{i}\right\}\right)=\left|\left\{B_{i}\right\}\right| /|M R|$ is the refinement degree of $\left\{B_{j}\right\}$.

Manufacturing resources can be divided into different results according to different manufacturing capability demands. Through a proper abstraction of manufacturing capability, a manufacturing task can easily find the eligible manufacturing resources among all the available ones. When $\operatorname{Gr}\left(\left\{B_{i}\right\}\right)=1,\left\{B_{j}\right\}$ is the finest division of $M R$. Manufacturing capabilities according to the finest division are called atomic capability. In the same way, manufacturing capabilities are called complex capability, which are according to the division with $\operatorname{Gr}\left(\left\{B_{i}\right\}\right)<1$.

Definition 2. A manufacturing resource is represented as a triple, MResource::= (MR_Id, NFeature, FFeature).

(1) MR_Id is the identification of the manufacturing resource. It can be represented by the Uniform Resource Identifier of the manufacturing resource.

(2) NFeature represents the nonfunctional features of the manufacturing resource, which can be used to efficiently evaluate the resource organization, storage, management, and utilization. Furthermore, it can be divided into static information and dynamic 
information. NFeature::=(StaticInfo, DynamicInfo). Static information is relatively stable in manufacturing resource lifecycles, such as resource type, resource structure, resource provider etc. Dynamic information changes over time, such as resource state, resource inventory, resource planning etc.

(3) FFeature presents functional features of the manufacturing resource, which are equivalence to the inherent manufacturing capabilities of the manufacturing resource. The detail information of each functional feature is consisted of three parts. FFeature:: $=\left\{\left(B_{j}\right.\right.$, CapaInfo, CstrInfo $\left.)\right\} . B_{j}$ is the manufacturing resource granular which is dependent on the manufacturing demands. CapaInfo represents the operations included in the corresponding manufacturing capability. CstrInfo is the specific constraint information of the manufacturing capability. Fig.3 illustrates an example of the manufacturing resource with multi-granularity capabilities.

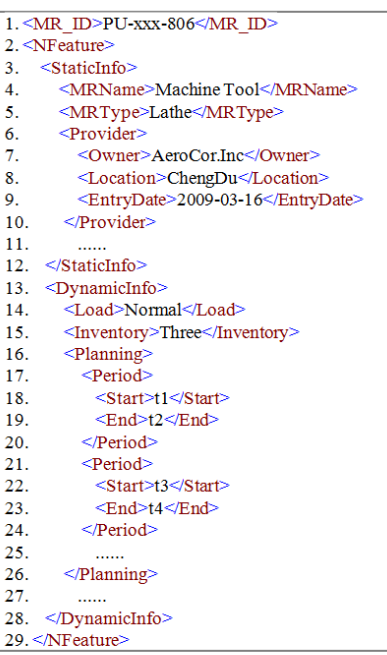

Nonfunctional Features

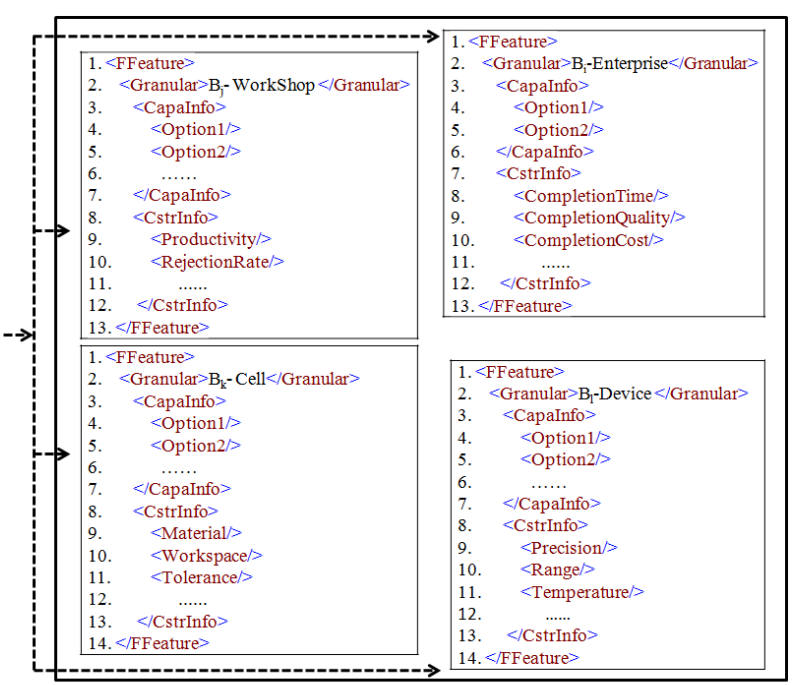

Functional Features with multi-granularity

Fig. 3. An excerpt of a manufacturing resource description

The described manufacturing resource are included in four different manufacturing resource granular, which are on enterprise level, workshop level, cell level, and device level respectively. For example, the manufacturing capability in the manufacturing resource granular on device level can be described from process type, geometrical characteristic, speed etc. Accordingly, constraints for the manufacturing capability are related to parameters such as processing precision, part range, processing temperature etc. Each resource can be viewed as the combination of nonfunctional features and functional features. Based on object-oriented methodology, each resource inherits all attributes from its superclass and can have its own new attributes on demand. By the flexibility and openness of the proposed description model, the templates of each special kind of manufacturing resources can be customized. 


\subsection{Manufacturing Cloud Service Encapsulation Using Extended OWL-S}

OWL-S is a web ontology language for web services which supports the dynamic services discovery, invocation, composition and mediation. A complete description of web service capabilities can be deployed through three modules, service profile, service model, and service grounding. However, OWL-S is insufficient for manufacturing cloud services because manufacturing cloud services are different from traditional web services. A web service is applied to the software development. It transforms information entities. While a manufacturing cloud service is associated with manufacturing equipment. It transforms the raw materials into physical objects with specific geometry. The capabilities of a web service are associated with input, output, precondition, effect. While manufacturing capabilities contain a variety of complex information, such as processing object, available product etc. Furthermore, information of manufacturing capabilities must be as precise and rich as possible. For example, a web service requestor only concerns about the QoS indicators. The invocation and composition of manufacturing cloud services are more complex. It is related to many factors, such as service location, service state. Therefore, in addition to the QoS indicators are needed to expose to the requestor, performance indicators of manufacturing cloud services are also needed to be described.

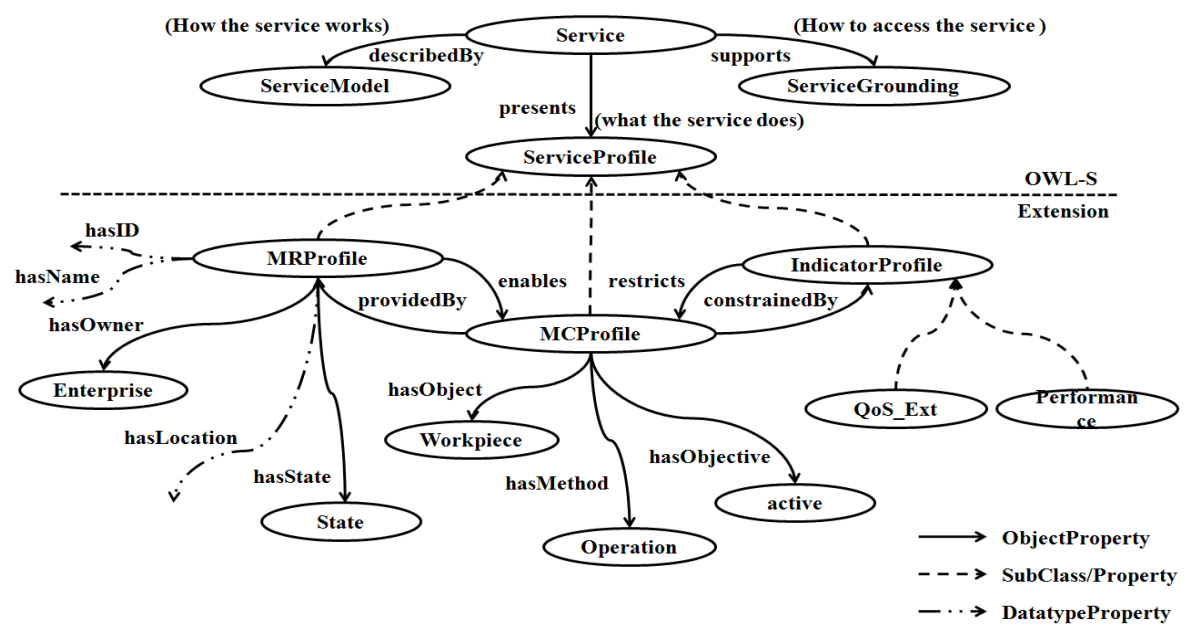

Fig. 4. Ontology model by extending OWL-S

An effective virtualization method requires formalized and structured representations of manufacturing resources. The information of manufacturing capabilities and their constraints plays an important role in manufacturing service discovery and matchmaking. This paper extends OWL-S by defining three classes in order to represent the indispensable information of manufacturing cloud services. As shown in Fig.4, the extended ontology model concerns the issues and elements related to collaborative manufacturing, such as available raw materials or castings, available equipment, available products, resource state, and various performance indicators 
relevant to manufacturing capabilities. MRProfile, MCProfile, and IndicatorProfile are the subclasses of ServiceProfile. MRProfile represents the nonfunctional information of the entities that enables manufacturing capabilities. MCProfile describes the functionalities of the manufacturing resource. It gives a detailed description of how the manufacturing cloud service operates. Manufacturing capabilities can be divided into atomic capability and complex capability. Complex capabilities are combinations of atomic capabilities, usually formed by a combination of devices, such as a machine tool, fixture, and a worker. IndicatorProfile presents constraint information from both providers and requestors perspectives.
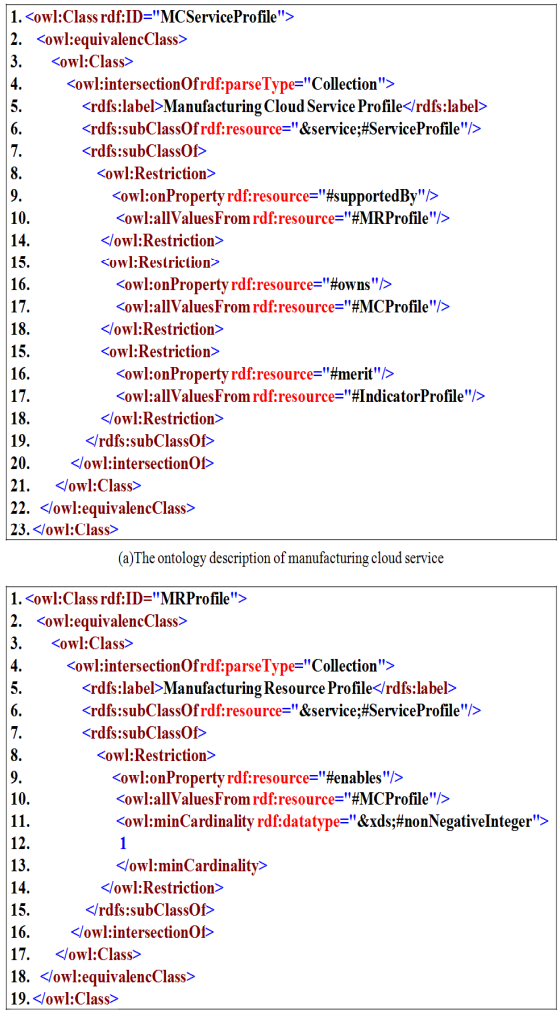

(c) The ontology description of manufacturing resource
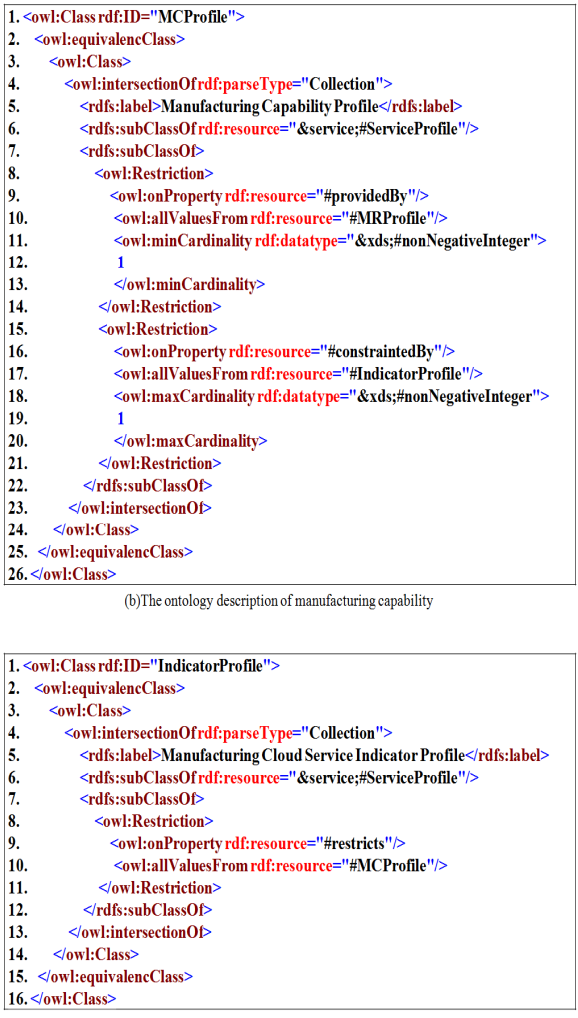

(d)The ontology description of indicator

Fig. 5. The OWL schema for core concepts in ontology model

The description of the core concepts are given in Fig.5 by OWL. Manufacturing resources and manufacturing capabilities are the general objects consisting of manufacturing cloud services. Manufacturing resources enable various manufacturing capabilities while manufacturing capabilities are provided by different manufacturing resources. They have multiple to multiple mapping relationship. In this paper, both 
manufacturing resources and manufacturing capabilities cannot exist independently. A manufacturing resource enables at least one manufacturing capability. In the same way, a manufacturing capability is provided by at least one manufacturing resource. The indicator information enhances the possibility and accuracy of manufacturing cloud service discovery. The three extended classes form the core concepts of ontology model for manufacturing cloud services. Concepts of relevant product and resource elements needed for matchmaking are also formalized defined.

\section{Case Study}

\subsection{Background}

The proposed method is demonstrated by a manufacturing resource in a big aerospace company of China, which owns its core competence with coordinating partners include designers, suppliers, and testers in various fields. Various manufacturing resources are distributed among partners. Because of the importer virtualization for resources, existing collaborative manufacturing operational forms could not be directly used to cloud manufacturing environments. We implemented the proposed virtualization method and applied it to the aerospace company to promote its manufacturing resources and capabilities sharing in cloud manufacturing.

\subsection{The Description of Manufacturing Cloud Services}

To illustrate the significance of the proposed method, this paper gives the description of a manufacturing cloud service which has an atomic manufacturing capability of "joining" on device level, as depicted in Fig.6. The manufacturing capability is provided by a joining machine. The document on the left side is described based on OWL-S and the document on the right side is described based on the proposed method. The code lines are numbered to facilitate discussion. Obviously they are not a part of the service description.

In the description of manufacturing cloud service based on OWL-S, the information of both joining machine and joining capability are included in the same profile, such as Lines 10-25 in the figure 6(a). This means that the manufacturing resource and its manufacturing capability are tightly coupled with each other. That is not conductive to deal with the matchmaking between manufacturing resources and manufacturing tasks on different collaboration levels. When the manufacturing resource unexpectedly exits cloud manufacturing systems, or it suddenly stops running for some reasons, the manufacturing cloud service cannot be invoked normally. From another perspective, the information is not sufficient to represent complex manufacturing capabilities from both structure and constraints aspects. Manufacturing requestors cannot find their interest information in the document. As a result, the resource utilization of the cloud manufacturing systems is reduced. 


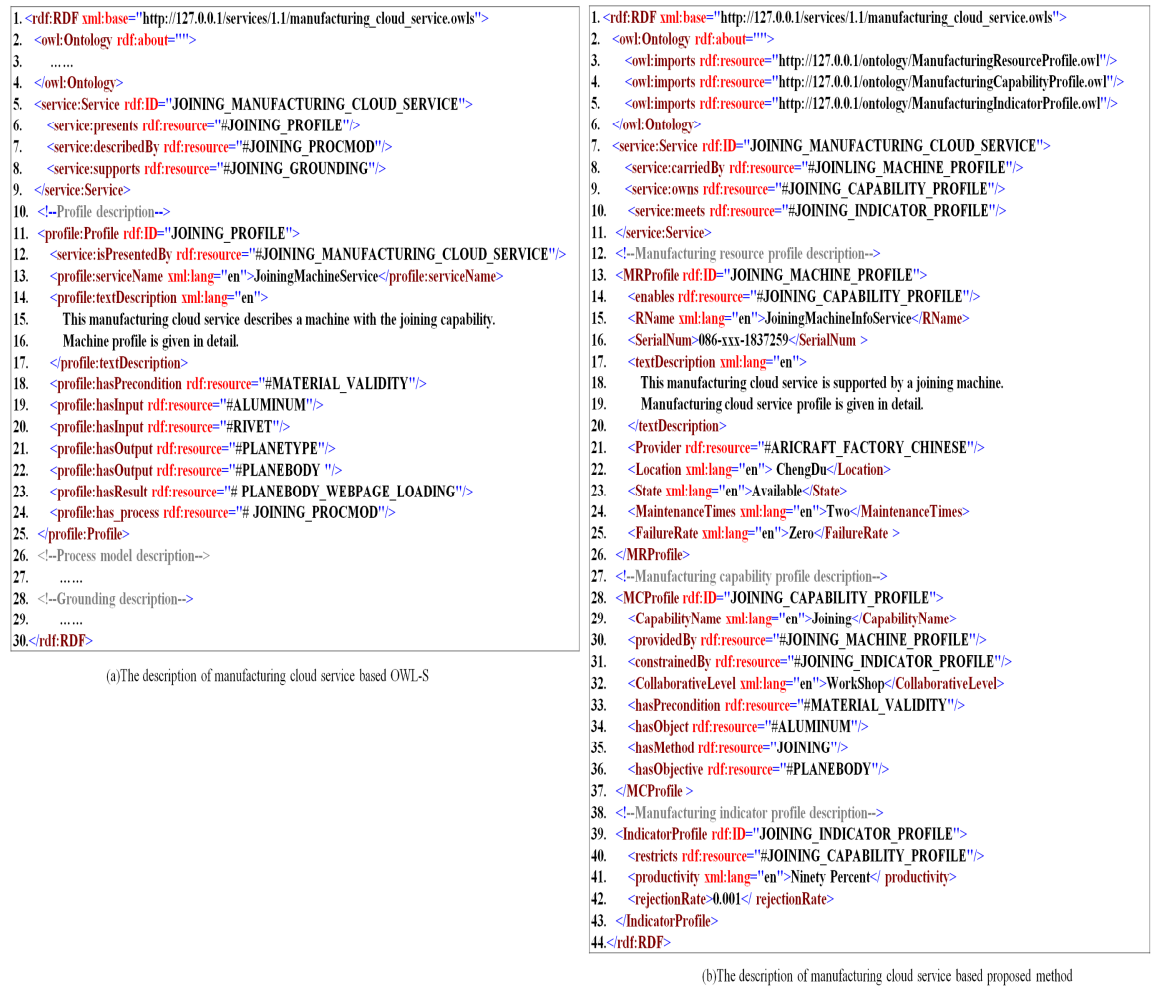

Fig. 6. The comparison of manufacturing cloud service description

In the description of manufacturing cloud service based on the proposed method, the information of manufacturing resource, manufacturing capability and performance indicators are described separately. In figure 6(b), Lines 1-6 specify the basis of XML coding and the namespaces declaration of the ontologies exploited by the description of the manufacturing cloud service. Lines 7-11 introduce a description of the manufacturing cloud service, identified as JOINING_MANUFACTURING_CLOUD _SERVICE, showing the relationship between manufacturing cloud service and the core concepts in the extended ontology model. Lines 12-26 focus on the manufacturing resource description. The manufacturing resource profile, named JOINING_MACHINE_PROFILE provides the resource name, the serial number, a textual description, the provider, the location, the state, the maintenance times, the failure rate and the associated capability. Lines 27-37 focus on the manufacturing capability description. The manufacturing capability profile, named JOINING_ CAPABILITY_PROFILE provides the capability name, the capability provider, the performance indicator, the capability level, the precondition, the processing object, the method, and the objective. Lines 38-43 present indicator profile description. The indicator profile named JOINING_INDICATOR_PROFILE provides the productivity, the rejection rate. The information is the performance indicators when the manufacturing resource provides the manufacturing capability. In other words, the 
capability is provided by the manufacturing resource named JOINING_MACHINE_ PROFILE and takes material "aluminum" as processing object and returns the plane body. As precondition, the capability checks the validity of the material, whereas as result, it completes the product with specific performance. By this way, manufacturing capabilities are artificially decoupled with manufacturing resources to support agile and flexible resource discovery and sharing.

\subsection{Discussion}

The proposed method and OWL-S present several similarities and differences. Like the OWL-S, the proposed method describes manufacturing cloud services from semantic perspective. Both of them aim to contribute to service discovery, matching, and composition automatically. However, unlike OWL-S, which represents web service capabilities in the service profile, the proposed method describes manufacturing cloud services based on three profiles. The description of multigranularity manufacturing capabilities and related performance indicators are more suitable to the requirements of the requestors. By this way, an effective and efficiency matching approach can be developed. Manufacturing resources can be easily discovered by demands coming from different collaborative levels in two steps. Firstly, based on manufacturing capabilities, the product requirement can rapidly select a set of manufacturing resource candidates. Then based on the performance indicators, the best service among the candidate services can be determined.

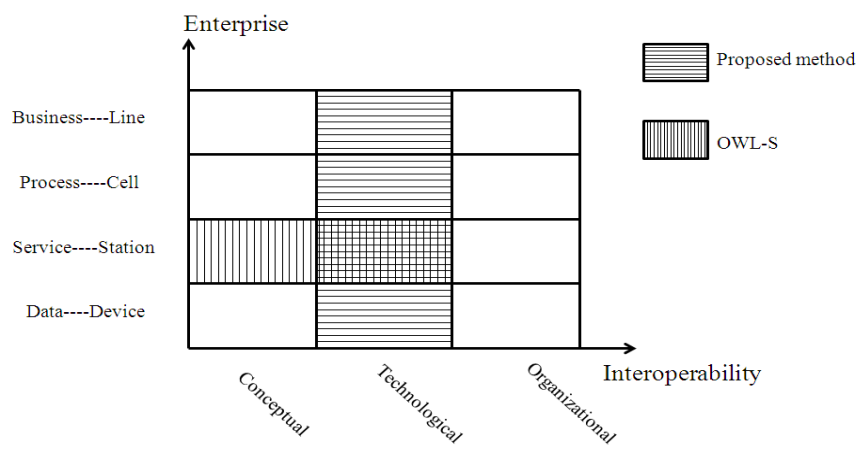

Fig. 7. The comparison from enterprise interoperability perspective

From enterprise interoperability point of view, Chen et al. [13] proposed an enterprise interoperability framework with two basic dimensions, as shown in Fig.7. Enterprise dimension represents enterprise levels(business, process, service, data), which has the similar meaning of collaborative levels in cloud manufacturing. Interoperability dimension represents interoperability barriers (conceptual, technological, organizational). The intersection of a level category (row) and a barrier category(column) constitutes an interoperability sub-domain. The proposed method contributes to remove technological barrier on various levels of enterprise dimension, 
while OWL-S addresses the conceptual and technological barriers on service level. Knowledge aspect is not explored in depth by the proposed method. In order to manage distributed manufacturing resources, a semantic manufacturing resource meta-model can be designed based on the core concepts proposed in this paper.



Fig. 8. The significance from collaborative point view

From enterprise collaboration point of view, the proposed method provides a feasible solution to enhance the competitive advantages of the collaborative networks. As shown in Fig.8, the multi-granularity manufacturing resource model includes comprehensive information on the phases of product development. Information views can be derived from the model to support various manufacturing applications. Manufacturing enterprises can achieve their business objectives in a time, quality and cost effective manner.

\section{Conclusions}

This paper presents an effective resource virtualization mechanism for cloud manufacturing. A three layers virtualization framework is proposed to transform distributed manufacturing resources into manufacturing cloud services. By analyzing the features of manufacturing resources and the granularity requirements of manufacturing capabilities, a multi-granularity manufacturing resource model is described. Manufacturing capabilities are encapsulated as manufacturing cloud services by extending OWL-S with three classes. Instead of building a centralized manufacturing resources repository, the multi-granularity manufacturing resource model manages manufacturing resources based on manufacturing capabilities. During the process of collaborative manufacturing, manufacturing resources are changeable while manufacturing capabilities are relatively sustained. Therefore, the proposed mechanism is flexible and scaleable because manufacturing cloud services are decoupled with manufacturing resources. In addition, manufacturing resources can be located and obtained according to multi-granularity manufacturing capabilities and related constraints. That contributes to support dynamic and automatic services discovery and composition.

Acknowledgment. This work was supported by National Natural Science Foundation of China under Grant 61070160. 


\section{References}

1. Xu, X.: From cloud computing to cloud manufacturing. Robotics and Computer-Integrated Manufacturing 28, 75-86 (2012)

2. Laguionie, R., Rauch, M., Hascoet, J.Y., Suh, S.H.: An extended manufacturing integrated system for feature-based manufacturing with STEP-NC. International Journal of Computer Integrated Manufacturing 24, 785-799 (2011)

3. Vichare, P., Nassehi, A., Kumar, S., Newman, S.T.: A unified manufacturing resource model for representing CNC machining systems. Robotics and Computer-Integrated Manufacturing 25, 99-1007 (2009)

4. Shen, W.M., Hao, Q., Wang, S.Y., Li, Y.S., Ghenniwa, H.: An agent-based serviceoriented integration architecture for collaborative intelligent manufacturing. Robotics and Computer-Integrated Manufacturing 23, 315-325 (2007)

5. Liang, F., Fung, R.Y.K., Jiang, Z.: Modeling approach and behavior analysis of manufacturing resources in virtual cellular manufacturing systems using resource element concept. International Journal of Computer Integrated Manufacturing 24, 1168-1182 (2011)

6. Zhang, W.Y., Cai, W., Qiu, J., Yin, J.W.: Managing distributed manufacturing knowledge through multi-perspective modeling for semantic web applications. International Journal of Production Research 22, 1144-1153 (2009)

7. Shi, S.Y., Mo, R., Yang, H.C., Chang, Z.Y., Chen, Z.F.: An implement of modeling resource in a manufacturing grid for resource sharing. International Journal of Computer Integrated Manufacturing 20, 169-177 (2007)

8. Wang, P.W., Jin, Z., Liu, L., Cai, G.Q.: Building towards capability specifications of web services based on an environment ontology. IEEE Transactions on Knowledge and Data Engineering 20, 547-561 (2008)

9. Jang, J., Jeong, B., Kulvatunyou, B., Chang, J., Cho, H.: Discovering and integrating distributed manufacturing services with semantic manufacturing capability profiles. International Journal of Computer Integrated Manufacturing 21, 631-646 (2008)

10. Ameri, F., Dutta, D.: A matchmaking methodology for supply chain deployment in distributed manufacturing environments. Journal of Computing and Information Science in Engineering 8, 001002:1-001002:9 (2008)

11. Atzori, L., Iera, A., Morabito, G.: The internet of things: a survey. Computer Networks 54, 2787-2805 (2010)

12. Yan, X., Lau, R.Y.K., Song, D., Li, X., Ma, J.: Toward a semantic granularity model for domain-specific information retrieval. ACM Transactions of Information Systems 29, Article 15 (2011)

13. Chen, D., Doumeingts, G., Vernadat, F.: Architectures for enterprise integration and interoperability: past, present, and furture. Computers in Industry 59, 647-659 (2008) 